PROCEEDINGS OF THE

AMERICAN MATHEMATICAL SOCIETY

Volume 138, Number 12, December 2010, Pages 4373-4385

S 0002-9939(2010)10434-3

Article electronically published on May 27, 2010

\title{
SYMBOLIC DYNAMICS FOR NONHYPERBOLIC SYSTEMS
}

\author{
DAVID RICHESON AND JIM WISEMAN
}

(Communicated by Bryna Kra)

\begin{abstract}
We introduce index systems, a tool for studying isolated invariant sets of dynamical systems that are not necessarily hyperbolic. The mapping of the index systems mimics the expansion and contraction of hyperbolic maps on the tangent space, and they may be used like Markov partitions to generate symbolic dynamics. Every continuous dynamical system satisfying a weak form of expansiveness possesses an index system. Because of their topological robustness, they can be used to obtain rigorous results from computer approximations of a dynamical system.
\end{abstract}

\section{INTRODUCTION}

Hyperbolicity is one of the most important ideas in dynamical systems. Every hyperbolic diffeomorphism admits a Markov partition, i.e. a finite collection of rectangles that stretch or shrink in different directions and map nicely onto each other. The interaction of rectangles under a single application of the map is sufficient to generate symbolic dynamics, which in turn gives global information about the dynamical system.

In this paper we introduce index systems, a topological generalization of hyperbolicity and Markov partitions. Index systems are composed of finitely many index pairs, a fundamental object from Conley index theory. The mapping of the index pairs mimics the expansion and contraction of the Markov rectangles, and they may be used to generate symbolic dynamics.

The benefit of this topological approach is that it applies in much more general situations than do Markov partitions. Hyperbolicity is a strong condition (requiring at least a manifold and a differentiable map) that can be difficult to verify. However, index systems can be constructed on metric spaces in which the map is not differentiable. In particular, every discrete dynamical system that satisfies a weak form of expansiveness (one of the properties of every hyperbolic system) is guaranteed to have a nontrivial index system. Moreover, unlike Markov partitions, index systems are robust under slight perturbations of the map. This will enable us (in future work) to obtain rigorous results about dynamical systems from computer approximations. As we will see, however, the expense of this generality is that whereas a Markov partition produces a subshift of finite type, an index system generates a cocyclic subshift (see Section 5).

Received by the editors September 4, 2009 and, in revised form, February 9, 2010.

2010 Mathematics Subject Classification. Primary 37B30, 37B10; Secondary 37M99.

(C)2010 American Mathematical Society

Reverts to public domain 28 years from publication 
The paper is organized as follows. In Section 2 we recall necessary information about expansiveness and the Conley index. We discuss index systems and their properties in Section 3. We show how to use them to detect orbits of the dynamical system in Section 4 and how they can be used to generate symbolic dynamics in Section 5. In Section 6 we show that all maps with a weak form of expansiveness have index systems. In Section 7 we give examples.

We are grateful to the anonymous referee for valuable suggestions for this paper and future work.

\section{BACKGROUND}

Unless otherwise specified, throughout this paper we let every space $X$ be a compact metric space and every dynamical system $f: X \rightarrow X$ be a continuous map. An orbit of $f$ is a bi-infinite sequence $\left(x_{i} \in X: i \in \mathbb{Z}\right)$ with the property that $f\left(x_{i}\right)=x_{i+1}$ for all $i \in \mathbb{Z}$.

2.1. Expansiveness. A homeomorphism $f: X \rightarrow X$ is expansive if there exists $\rho>0$ such that for any distinct points $x, y \in X$, there is an integer $n$ with $d\left(f^{n}(x), f^{n}(y)\right)>\rho$. In other words, if the orbits of two points stay close together for all time, then they must be the same point. Expansiveness is a strong form of sensitive dependence on initial conditions since any two distinct points must eventually move apart in either forward or backward time. In practical terms, this means that any small initial measurement error will lead to large errors in predicting behavior. It is not difficult to show that if $S$ is a hyperbolic invariant set for a diffeomorphism $f$, then $f$ restricted to $S$ is expansive ([15, 26]).

Although expansiveness is defined as a metric property, on compact spaces it is independent of the metric (of those compatible with the topology), and there is a very simple and useful topological characterization. Moreover, the topological definition can be extended trivially to continuous maps. To state it we need some preliminary definitions.

We begin with the definition of an isolated invariant set, a notion that is also central to Conley index theory and will be discussed in more detail in Section 2.2 A set $S \subset X$ is an isolated invariant set for a continuous map $f: X \rightarrow X$ provided there is a compact set $I$ with $S=\operatorname{Inv} I \subset \operatorname{Int} I$ (where Inv $I$ denotes the maximal invariant subset of $I$ ). The set $I$ is called an isolating neighborhood for $S$. Examples of isolated invariant sets include hyperbolic periodic orbits, attractors, and the invariant Cantor set inside the Smale horseshoe.

For any dynamical system $f: X \rightarrow X$, let $f \times f: X \times X \rightarrow X \times X$ be the map $(f \times f)\left(x_{1}, x_{2}\right)=\left(f\left(x_{1}\right), f\left(x_{2}\right)\right)$. Let $1_{X}=\{(x, x) \in X \times X\}$ denote the diagonal of $X \times X$.

Definition 1. A continuous map $f: X \rightarrow X$ is expansive if the diagonal $1_{X} \subset$ $X \times X$ is an isolated invariant set with respect to $f \times f$.

Note that when $f$ is a homeomorphism this definition of expansive is equivalent to the metric definition ([1, Def. 11.4]).

We have defined expansiveness for a map $f: X \rightarrow X$. If $S$ is an isolated invariant subset of $X$ we say that $f$ is expansive on $S$ if $f$ restricted to $S$ is expansive. Note that this is equivalent to the condition that the set $1_{S}=\{(x, x): x \in S\} \subset X \times X$ is an isolated invariant set for $f \times f: X \times X \rightarrow X \times X$ ([1, Ex. 11.5]). 
2.2. Conley index. The discrete Conley index is a powerful topological tool for studying isolated invariant sets. Roughly speaking, the Conley index assigns to each isolated invariant set for $f$ a pointed topological space and a base-point preserving map on it, $f_{P}$, which is unique up to an equivalence relation. By studying the simpler map $f_{P}$ we can draw conclusions about the original map $f$. Our discussion of the discrete Conley index is based on that in [11, where one can find more details and proofs of the theorems below.

Ideally one would like to place an isolated invariant set, $S$, between levels of a filtration. In other words, we would like sets $N_{0} \subset N_{1}$, both of which map into their interiors, such that $S=\operatorname{Inv}\left(N_{1} \backslash N_{0}\right)$. In practice this may not be possible. Instead we must settle for a topological pair that behaves locally like a filtration.

Definition 2. Let $S$ be an isolated invariant set and suppose $L \subset N$ are compact sets. The pair $(N, L)$ is an index pair for $S$ provided $N$ and $L$ are each the closures of their interiors and

(1) $\operatorname{cl}(N \backslash L)$ is an isolating neighborhood for $S$,

(2) $L$ is a neighborhood of the exit set, $N^{-}=\{x \in N: f(x) \notin \operatorname{Int} N\}$, in $N$, and

(3) $f(L) \cap \operatorname{cl}(N \backslash L)=\emptyset$.

The definition of an index pair given above was introduced in [11] (where they were called filtration pairs). This definition is similar to those in [4, 10, 21, 27, 25]. We see examples of index pairs in Figure 1.
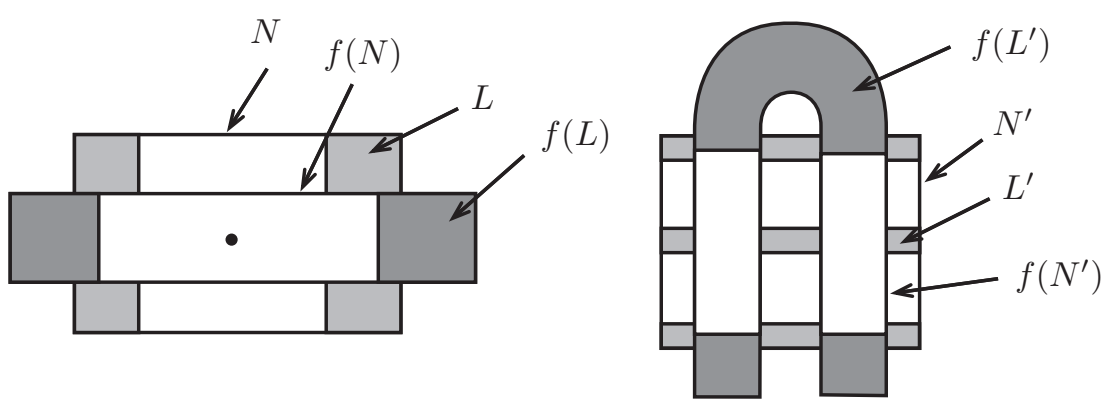

Figure 1. Index pairs: $(N, L)$ for a fixed point saddle, $\left(N^{\prime}, L^{\prime}\right)$ for the horseshoe Cantor set, and their images under $f$.

Given a neighborhood $U$ of an isolated invariant set $S$ there exists an index pair $P=(N, L)$ with $N \backslash L \subset U$. Given such an index pair we form the pointed space $N_{L}$ by collapsing $L$ to a point, $[L]$ (see Figure 2). Thus $f$ induces a continuous map $f_{P}: N_{L} \rightarrow N_{L}$. The base point $[L]$ is an attracting fixed point of $f_{P}$. The choice of index pairs for $S$ is not unique, and different choices can lead to topologically different maps $f_{P}$. However, the choice is unique up to shift equivalence (we will use $\cong$ to denote shift equivalence). The resulting equivalence class is called the homotopy Conley index. For a definition of shift equivalence and a proof of the facts in this paragraph, see [11.

In practice, one may wish to compute the relative homology (or cohomology) of the pointed space and the induced map $\left(f_{P}\right)_{*}: H_{*}\left(N_{L},[L]\right) \rightarrow H_{*}\left(N_{L},[L]\right)$. While homotopy carries more information than homology, homology is often much 

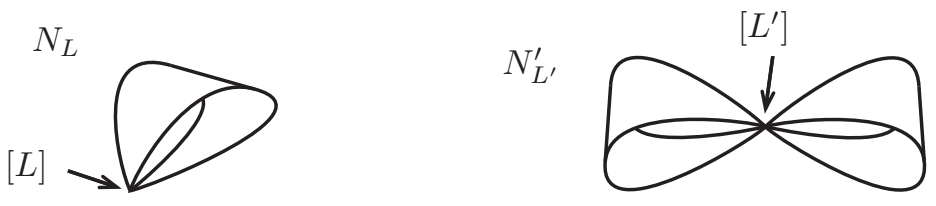

Figure 2. The pointed spaces $N_{L}$ and $N_{L^{\prime}}^{\prime}$ obtained from the filtration pairs for the saddle and the horseshoe.

simpler to work with, particularly in a computational setting. Again, the map $\left(f_{P}\right)_{*}$ is unique up to shift equivalence. We call this equivalence class the homology Conley index, $\operatorname{Con}_{*}(S)$. (Note: we may apply the Leray functor to $\left(f_{P}\right)_{*}$ to obtain an automorphism of a graded group, which is an invariant for $S$ (see 21]).)

For instance, the fixed point saddle in Figure 1 has $\operatorname{Con}_{*}(S) \cong(\mathbb{Z}$, Id), where the $\mathbb{Z}$ is in dimension 1. The invariant Cantor set in the horseshoe has $\operatorname{Con}_{*}(S) \cong 0$ (from a homological perspective the two arms of the horseshoe cancel one another). Note that that if $\operatorname{Con}_{*}(S) \neq 0$, then $S \neq \emptyset$ (this is called Ważewski's theorem), but the horseshoe shows that the converse is not true.

An important feature of the Conley index is that if $I$ is an isolating neighborhood for $f$, then $I$ is also an isolating neighborhood for $\operatorname{Inv}(I, g)$ for any $g$ that is $C^{0}$-close to $f$, and in this case $\operatorname{Inv}(I, f)$ and $\operatorname{Inv}(I, g)$ have the same Conley index ([11]). This robustness allows one to compute the Conley index from a suitable numerical approximation of the map ([16, 19, 22, 23).

\section{INDEX SYSTEMS}

For differentiable maps it is frequently useful to look at the behavior of orbits on the manifold and also the behavior of the derivative on the tangent space following these orbits. Indeed, this is precisely what one does with hyperbolicity. As we see in this section, for an expansive map $f: X \rightarrow X$ we may use $f \times f: X \times X \rightarrow X \times X$ to obtain a nice analogue of the differentiable situation. We will see that slices of a neighborhood $N$ of the diagonal (that is, intersections of $\{x\} \times X$ with $N$ ) are the analogues of the tangent spaces, and it is from these slices that we build the index system.

Suppose $\mathcal{I}=\left(I_{i}: i \in \mathbb{Z}\right)$ is a sequence of compact sets in $X$ (in practice $\left\{I_{i}: i \in \mathbb{Z}\right\}$ will be a finite set of sets). We say that an orbit $\left(x_{i}\right)$ follows $\mathcal{I}$ if $x_{i} \in I_{i}$ for all $i \in \mathbb{Z}$. We call such a sequence, $\mathcal{I}$, an isolating neighborhood chain if for any orbit $\left(x_{i}\right)$ that follows $\mathcal{I}, x_{i} \in \operatorname{Int}\left(I_{i}\right)$ for all $i \in \mathbb{Z}$. For $\mathcal{I}$ an isolating neighborhood chain, let $\operatorname{Inv}_{0}(\mathcal{I}) \subset \operatorname{Int}\left(I_{0}\right)$ denote the set of points whose orbits follow $\mathcal{I}$; that is,

$$
\operatorname{Inv}_{0}(\mathcal{I})=\left\{x \in I_{0}: \text { there exists an orbit }\left(x_{i}\right) \text {, with } x_{0}=x \text {, that follows } \mathcal{I}\right\} .
$$

Note that $\operatorname{Inv}_{0}(\mathcal{I})$ is not, in general, $f$-invariant.

An index system is a collection of compact pairs. Each is similar to an index pair, but instead of necessarily mapping to itself under $f$, it maps to one or more of the pairs in the index system (see Figure 3). More precisely, we have the following definition.

Definition 3. An index system is a finite collection of compact pairs, $\mathcal{P}=\left\{P_{a}=\right.$ $\left.\left(N_{a}, L_{a}\right): a \in \mathcal{A}\right\}$, such that 


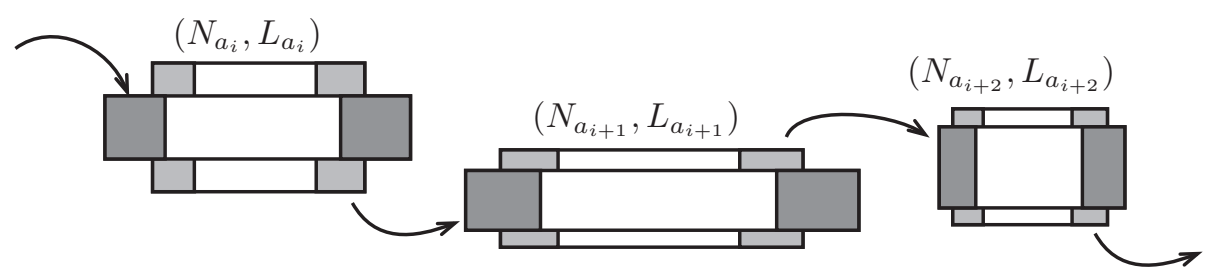

Figure 3. $\left(N_{a_{i}}, L_{a_{i}}\right)$ precedes $\left(N_{a_{i+1}}, L_{a_{i+1}}\right)$ which precedes $\left(N_{a_{i+2}}, L_{a_{i+2}}\right)$.

(1) for each $a \in \mathcal{A}$, there exists at least one $b \in \mathcal{A}$ such that $P_{a}$ precedes $P_{b}$, that is, such that

(a) $L_{a}$ is a neighborhood of the exit set, $N_{a b}^{-}=\left\{x \in N_{a}: f(x) \notin \operatorname{Int} N_{b}\right\}$, in $N$, and

(b) $f\left(L_{a}\right) \cap \operatorname{cl}\left(N_{b} \backslash L_{b}\right)=\emptyset$; and

(2) any sequence $\left(I_{i}=\operatorname{cl}\left(N_{a_{i}} \backslash L_{a_{i}}\right): P_{a_{i}}\right.$ precedes $P_{a_{i+1}}$ for all $\left.i \in \mathbb{Z}\right)$ is an isolating neighborhood chain.

For each $a$, we form the pointed space $N_{a L_{a}}$ by collapsing $L_{a}$ to a point, $\left[L_{a}\right]$. If $P_{a}$ precedes $P_{b}$, then $f$ induces a continuous map $f_{a, b}: N_{a L_{a}} \rightarrow N_{b L_{b}}$.

We can think of an index system as a directed graph. Each pointed space $N_{a L_{a}}$ is a vertex, with an edge from $N_{a L_{a}}$ to $N_{b L_{b}}$ if $P_{a}$ precedes $P_{b}$. (The induced maps $f_{a, b}$ are analogues of the induced maps on the tangent bundle for differentiable systems.) We say that a finite or infinite sequence $\left(a_{i}\right)$ is allowable if it corresponds to a path in the graph, that is, if $P_{a_{i}}$ precedes $P_{a_{i+1}}$ for all $i$.

Given an index system $\mathcal{P}$, we define its invariant set by $\operatorname{Inv}(\mathcal{P})=\bigcup \operatorname{Inv}_{0}\left(\left(I_{i}=\right.\right.$ $\left.\operatorname{cl}\left(N_{a_{i}} \backslash L_{a_{i}}\right)\right)$ ), where the union is over all allowable sequences $\left(a_{i}: i \in \mathbb{Z}\right)$. Thus $\operatorname{Inv}(\mathcal{P})$ is the set of points on orbits following an allowable sequence of pairs. Let $\mathcal{P}$ be an index system. Define $\operatorname{Inv}^{m}(\mathcal{P})$ to be the set of $x$ such that there exists an orbit segment $\left(x_{i}\right)_{i=-m}^{m}$ with $x_{0}=x$ and $x_{i} \in \operatorname{cl}\left(N_{a_{i}} \backslash L_{a_{i}}\right)$ for some finite allowable sequence $\left(a_{i}\right)_{i=-m}^{m}$.

Lemma 4. Let $\mathcal{P}$ be an index system. Then $\operatorname{Inv}(\mathcal{P})=\bigcap_{m=0}^{\infty} \operatorname{Inv}^{m}(\mathcal{P})$.

Proof. The proof is based on that of [11, Prop. 2.2], which is the same result for index pairs instead of index systems. It is obvious that $\operatorname{Inv}(\mathcal{P}) \subset \bigcap_{m=0}^{\infty} \operatorname{Inv}^{m}(\mathcal{P})$. To prove the opposite inclusion, let $x$ be an element of $\bigcap_{m=0}^{\infty} \operatorname{Inv}^{m}(\mathcal{P})$; we must show that $x \in \operatorname{Inv}(\mathcal{P})$.

For an allowable sequence $\left(a_{-1}, a_{0}\right)$ with $x \in \operatorname{cl}\left(N_{a_{0}} \backslash L_{a_{0}}\right)$, define $X_{1}^{\left(a_{-1}, a_{0}\right)}=$ $f^{-1}(x) \cap \operatorname{cl}\left(N_{a_{-1}} \backslash L_{a_{-1}}\right)$. Then, for $\left(a_{-k}, \ldots, a_{0}\right)$ an allowable sequence with $x \in$ $\operatorname{cl}\left(N_{a_{0}} \backslash L_{a_{0}}\right)$, inductively define $X_{k}^{\left(a_{-k}, \ldots, a_{0}\right)}=f^{-1}\left(X_{k-1}^{\left(a_{-k+1}, \ldots, a_{0}\right)}\right) \cap \operatorname{cl}\left(N_{a_{-k}} \backslash L_{a_{-k}}\right)$. Each $X_{k}^{\left(a_{-k}, \ldots, a_{0}\right)}$ is compact and $f\left(X_{k}^{\left(a_{-k}, \ldots, a_{0}\right)}\right) \subset X_{k-1}^{\left(a_{-k+1}, \ldots, a_{0}\right)}$. Set $X_{k}=$ $\bigcup X_{k}^{\left(a_{-k}, \ldots, a_{0}\right)}$, where the union is over all allowable sequences $\left(a_{-k}, \ldots, a_{0}\right)$ with $x \in \operatorname{cl}\left(N_{a_{0}} \backslash L_{a_{0}}\right)$; then $X_{k}$ is a nonempty, compact set with $f\left(X_{k}\right) \subset X_{k-1}$.

Now define $Y_{k}=\bigcap_{n \geq 1} f^{n+1}\left(X_{n+k}\right)$. As the intersection of a nested sequence of nonempty compact subsets of $X_{k}, Y_{k}$ is a compact, nonempty subset of $X_{k}$, so $f\left(Y_{1}\right)=\{x\}$ and for $k>1$,

$$
f\left(Y_{k}\right)=\bigcap_{n \geq 1} f^{n+1}\left(X_{n+k}\right)=\bigcap_{n \geq 2} f^{n}\left(X_{n+k-1}\right)=Y_{k-1} .
$$


Finally, define $x_{-1}$ to be any point of $Y_{1}$, and inductively define $x_{-k}$ to be any point in $Y_{k}$ with $f\left(x_{-k}\right)=x_{-k+1}$. If we set $x_{k}=f^{k}(x)$ for $k \geq 0$, then $\left(x_{i}\right)_{i=-\infty}^{\infty}$ is an orbit following an allowable sequence, so $x \in \operatorname{Inv}(\mathcal{P})$.

The following result follows immediately from Lemma 4 ,

Proposition 5. If $\mathcal{P}$ is an index system, then $\operatorname{Inv}(\mathcal{P})$ is a compact invariant set.

Definition 6. Let $S \subset X$ be an isolated invariant set. An index system $\mathcal{P}$ is an index system for $S$ if $\operatorname{Inv}(\mathcal{P})=S$.

In Section 6 we present situations in which we can guarantee the existence of index systems, and we give a procedure for constructing them that could be implemented on a computer.

\section{Detecting orbits}

In this section we show how to prove the existence of an orbit following a given allowable sequence of sets $\left(\operatorname{cl}\left(N_{a_{i}} \backslash L_{a_{i}}\right)\right)$. If $f$ had the shadowing property and the sets $\operatorname{cl}\left(N_{a_{i}} \backslash L_{a_{i}}\right)$ were small enough, then we could guarantee the existence of an orbit that follows this sequence $([26, \S 9.3])$. However, the shadowing property is difficult to verify in practice. Instead, we use the Conley index to verify that there is an $f$-orbit that "shadows" the sequence in the sense that each iterate is in the appropriate set $\operatorname{cl}\left(N_{a_{i}} \backslash L_{a_{i}}\right)$.

An allowable sequence $\left(a_{i}\right)$ yields a directed system

$$
\cdots \longrightarrow H_{*}\left(P_{a_{i}}\right) \stackrel{f_{a_{i}, a_{i+1}}}{\longrightarrow} H_{*}\left(P_{a_{i+1}}\right) \stackrel{f_{a_{i+1}, a_{i+2}}}{\longrightarrow} H_{*}\left(P_{a_{i+2}}\right) \longrightarrow \cdots
$$

The sequence $\left(P_{a_{i}}\right)$ has a nonzero orbital Conley index if any finite composition $f_{a_{n-1}, a_{n} *} \circ \cdots \circ f_{a_{m+1}, a_{m+2} *} \circ f_{a_{m}, a_{m+1} *}$ is nonzero (note that if we take coefficients in a field and $H_{*}\left(P_{a}\right)$ is finitely generated for each $a$, then the maps are simply matrices).

We have the following theorem, which is the orbital analogue of Ważewski's theorem.

Theorem 7. Let $\mathcal{P}$ be an index system for the isolated invariant set $S$, and let $\left(a_{i}\right)$ be an allowable sequence. If $\left(P_{a_{i}}\right)$ has a nonzero orbital Conley index, then there is an orbit in $S$ following $\left(\operatorname{cl}\left(N_{a_{i}} \backslash L_{a_{i}}\right)\right)$. If $f$ is expansive and the diameters of the sets $\operatorname{cl}\left(N_{a} \backslash L_{a}\right)$ are sufficiently small for all a, then this orbit is unique.

Proof. If the set $\bigcap_{i=-m}^{m} f^{-i}\left(\operatorname{cl}\left(N_{a_{i}} \backslash L_{a_{i}}\right)\right)$ were empty, then the induced map would send every point to the base point, and the corresponding map on homology would be zero. Since $\left(P_{a_{i}}\right)$ has a nonzero orbital Conley index, the set $\bigcap_{i=-m}^{m} f^{-i}\left(\operatorname{cl}\left(N_{a_{i}} \backslash L_{a_{i}}\right)\right)$ is nonempty for all $m$. The same argument used to prove Lemma 4 then shows that there is an orbit in $S$ following $\left(\operatorname{cl}\left(N_{a_{i}} \backslash L_{a_{i}}\right)\right)$.

Suppose $f$ is expansive on $S$ and that $\left(x_{i}\right)$ and $\left(y_{i}\right)$ are two orbits following $\left(\operatorname{cl}\left(N_{a_{i}} \backslash L_{a_{i}}\right)\right)$. Because $f$ is expansive, $1_{S}$ is an isolated invariant set for $f \times f$ with some isolating neighborhood $I$. Assuming the diameters of $\operatorname{cl}\left(N_{a_{i}} \backslash L_{a_{i}}\right)$ are small enough, that means that the $(f \times f)$-orbit $\left(\left(x_{i}, y_{i}\right)\right)$ remains in $I$ for all $i$. But this implies that for all $i,\left(x_{i}, y_{i}\right) \in 1_{S}$, or equivalently $x_{i}=y_{i}$. 


\section{Symbolic DYNAMiCS}

Many authors have used the Conley index or related techniques (for example, Easton's windows (9])) to detect symbolic dynamics; an incomplete list of references is [3, 5, 6, 8, 9, 12, 13, 14, 20, 22, 28, 29, 30, 31, 32, 33, 34. Most treatments resemble index systems, except that all of the index pairs are pairwise disjoint. This is an important difference: disjoint index pairs make the conclusions stronger, but they are significantly less useful because they cover less of the space. In 12, Gidea developed a notion, similar to index systems, of an orbital Conley index for compact sets traveling within a prescribed sequence of neighborhoods, which he applied to detect periodic orbits and symbolic dynamics. An important difference is that index systems are used to study invariant sets, while Gidea's orbital Conley index applies to noninvariant sets that travel along a bi-infinite sequence of boxes. In addition, there is no result similar to Theorem 9 for constructing the neighborhood sequence for Gidea's orbital Conley index, and the method for detecting symbolic dynamics requires that the neighborhoods be pairwise disjoint. As we see in this section, we can relax this requirement with index systems.

The mapping of rectangles in a Markov partition generates a directed graph, and paths through this graph become the elements of a subshift of finite type. The elements of this subshift can be paired uniquely with points in the dynamical system. The situation for index systems is similar, but more complicated. Intuitively, we would like allowable sequences from our directed graph of pointed spaces to generate symbolic dynamics. However, we must use the Conley index (as in Section 4) to determine when there is a true orbit corresponding to this allowable sequence, and then we must show that each point corresponds to only one symbol sequence.

In order to put this in proper context we must introduce the notion of a cocyclic subshift, a generalization of sofic shifts and subshifts of finite type. See [17] for more information about cocylic subshifts and [5, 30, 31 for examples of cocyclic subshifts in other dynamical and Conley index contexts.

Definition 8. Let $G$ be a directed graph with vertices $\{1, \ldots, n\}$ and at most one edge from any vertex to any other. Assign to each vertex $i$ a vector space $V_{i}$ and to the edge from $i$ to $j$ a linear transformation $T_{i, j}: V_{i} \rightarrow V_{j}$. The cocyclic subshift associated to $G$ consists of all words $\left(\ldots, \omega_{i-1}, \omega_{i}, \omega_{i+1}, \ldots\right)$ such that

(1) there is an edge from vertex $\omega_{i}$ to $\omega_{i+1}$ for all $i$, and

(2) any finite composition $T_{\omega_{i+m-1}, \omega_{i+m}} \circ \cdots \circ T_{\omega_{i}, \omega_{i+1}}$ is nonzero, together with the usual shift map.

An index system generates the cocyclic subshift corresponding to the graph with vertex set $\mathcal{A}$, vector spaces $H_{*}\left(P_{a}\right)$, edges from $a$ to $b$ if $P_{a}$ precedes $P_{b}$, and linear transformations $f_{a, b *}$. In other words, we take the homology of our directed graph of pointed spaces. At each vertex we place the homology of the given pair, $H_{*}\left(P_{a}\right)=H_{*}\left(N_{a L_{a}},\left[L_{a}\right]\right)$, and we label each directed edge from $H_{*}\left(P_{a}\right)$ to $H_{*}\left(P_{b}\right)$ with the corresponding induced map on homology $f_{a, b *}$. Then elements of the cocyclic subshift correspond to allowable sequences with nonzero orbital Conley index.

A further complication is that in general we do not get a conjugacy (or semiconjugacy) from $f$ to the cocyclic subshift because the sets $\left\{\operatorname{cl}\left(N_{a} \backslash L_{a}\right)\right\}$ are usually not pairwise disjoint. For example, say that the period-two word $(1,2,1,2, \ldots)$ is in the cocyclic subshift, which implies that there is a point $x \in X$ such that 
$x \in \operatorname{cl}\left(N_{1} \backslash L_{1}\right), f(x) \in \operatorname{cl}\left(N_{2} \backslash L_{2}\right), f^{2}(x) \in \operatorname{cl}\left(N_{1} \backslash L_{1}\right)$, and so on. But if the two sets are not disjoint, then $x$ could be a fixed point. The $\mathcal{P}$-itinerary of a point is not necessarily unique.

One way around this issue is simply to remove overlapping index pairs. That is, take a maximal subgraph of the cocyclic subshift graph consisting of vertices for which all of the corresponding sets $\operatorname{cl}\left(N_{a} \backslash L_{a}\right)$ are pairwise disjoint. Then, as desired, we will get a semi-conjugacy from an isolated invariant set in $X$ to the cocyclic subshift induced by the subgraph. The disadvantage of this approach is that we are losing information about $f$ when we throw away vertices.

Another approach is to look at powers $f^{n}$. In this case, only some, not all, of the index pairs must be disjoint in order to detect positive-entropy symbolic dynamics. For example, let $\omega=\left(\omega_{0}, \ldots, \omega_{n}\right)$ be a word in the cocyclic subshift, and define $S_{\omega} \subset X$ to be the subset $\operatorname{Inv}\left(\left\{x \in X: f^{i}(x) \in \operatorname{cl}\left(N_{\omega_{i}}\right), 0 \leq i \leq n\right\}, f^{n+1}\right)$. If, say, the sets $\operatorname{cl}\left(N_{1} \backslash L_{1}\right)$ and $\operatorname{cl}\left(N_{3} \backslash L_{3}\right)$ are disjoint, then the sets $S_{(1,1,1)}$ and $S_{(1,2,3)}$ are disjoint (even if $\operatorname{cl}\left(N_{1} \backslash L_{1}\right), \operatorname{cl}\left(N_{2} \backslash L_{2}\right)$, and $\operatorname{cl}\left(N_{3} \backslash L_{3}\right)$ are not pairwise disjoint), so we can define symbolic dynamics and get a semiconjugacy for $f^{3}$.

Using either method for a generic index system, we get only a semiconjugacy from the invariant set to the cocyclic subshift. Thus, while we can get a lower bound for the topological entropy of $f$, we cannot conclude that a periodic word for the subshift actually corresponds to a periodic point for $f$. However, if $f$ is expansive and the index pairs are sufficiently small, then by the uniqueness of orbits guaranteed by Theorem 7, we do get a conjugacy. Even in some cases when we do not have expansiveness (in particular, if the homology is nontrivial on exactly one level), we can use a version of the Lefschetz fixed point theorem (28) instead of Theorem 7 to detect periodic points for $f$. For the sake of brevity, we omit the details.

There are further details on techniques for generating symbolic dynamics from index systems in 24 .

\section{EXISTENCE OF INDEX SYSTEMS}

As we have said, hyperbolic diffeomorphisms admit Markov partitions. In fact, homeomorphisms that are expansive and have the shadowing property also admit Markov partitions (2), but expansivity alone is not sufficient. Furthermore, even when they exist, Markov partitions can be difficult to construct.

One of the benefits of index systems is that under certain general circumstances their existence is guaranteed. Moreover, the proof is constructive; it requires only the ability to find a single index pair. There are computer algorithms that will do that (see [7, for example).

It is immediate that every isolated invariant set $S$ has at least a trivial index system: the system consisting of the single pair $(N, L)$, where $(N, L)$ is an index pair for $S$. In the case that $f$ is expansive, we can do better.

Theorem 9. Let $f$ be expansive on an isolated invariant set $S$. Then there exists an index system $\mathcal{P}=\left\{\left(N_{a}, L_{a}\right)\right\}$ for $S$ of arbitrarily small diameter (that is, the diameter of $N_{a}$ is arbitrarily small for all a).

Proof. The idea of the proof is simple. Recall from Section 2.1 that since $S$ is expansive, the set $1_{S}=\{(x, x): x \in S\} \subset X \times X$ is an isolated invariant set for $f \times f$. Thus any neighborhood of $1_{S}$ contains an index pair $(N, L)$ for $1_{S}$ under $f \times f$. 
By taking cross-sections, we get pairs $\left(N_{x}, L_{x}\right)=\left(\pi_{2}((\{x\} \times X) \cap N), \pi_{2}((\{x\} \times\right.$ $X) \cap L)$ ) of arbitrarily small diameter (where $\pi_{2}: X \times X \rightarrow X$ is the projection onto the second coordinate). We would like these pairs to make up the index system. However, an index system must be finite, and in general this construction may give us infinitely many pairs, one for each $x \in S$.

By essentially discretizing the space and using the robustness of the Conley index we can modify the original index pair $(N, L)$ to yield only finitely many cross-sections. Let $D$ be the metric on $X \times X$ given by $D\left((x, y),\left(x^{\prime}, y^{\prime}\right)\right)=$ $\max \left(d\left(x, x^{\prime}\right), d\left(y, y^{\prime}\right)\right)$. By the definition of an index pair, there exists an $\varepsilon>0$ such that $D\left(1_{S}, L\right)>\varepsilon, D((f \times f)(L), \operatorname{cl}(N \backslash L))>\varepsilon$, and $D\left(N^{-}, \operatorname{cl}(N \backslash L)\right)>$ $\varepsilon$. Pick $\delta<\varepsilon / 3$ such that for any points $(x, y)$ and $\left(x^{\prime}, y^{\prime}\right)$ within $\varepsilon$ of $N$, if $D\left((x, y),\left(x^{\prime}, y^{\prime}\right)\right)<\delta$, then $D\left((f \times f)(x, y),(f \times f)\left(x^{\prime}, y^{\prime}\right)\right)<\varepsilon / 3$.

Let $\left\{V_{i}\right\}$ be a finite collection of compact subsets of $X$ of diameter less than $\delta$ such that $\pi_{1}(N) \cup \pi_{2}(N) \subset \bigcup_{i} \operatorname{Int}\left(V_{i}\right)$. This gives a product cover of $N,\left\{V_{i j}=V_{i} \times V_{j}\right\}$. We now construct a new index pair for $1_{S},(\tilde{N}, \tilde{L})$, by setting $\tilde{N}=\bigcup_{\left\{i j: V_{i j} \cap N \neq \emptyset\right\}} V_{i j}$ and $\tilde{L}=\bigcup_{\left\{i j: V_{i j} \cap L \neq \emptyset\right\}} V_{i j}$. (See Figure 4.) The set of cross-sections of $(\tilde{N}, \tilde{L})$ is finite, and it is straightforward to check that it forms an index system for $S$.
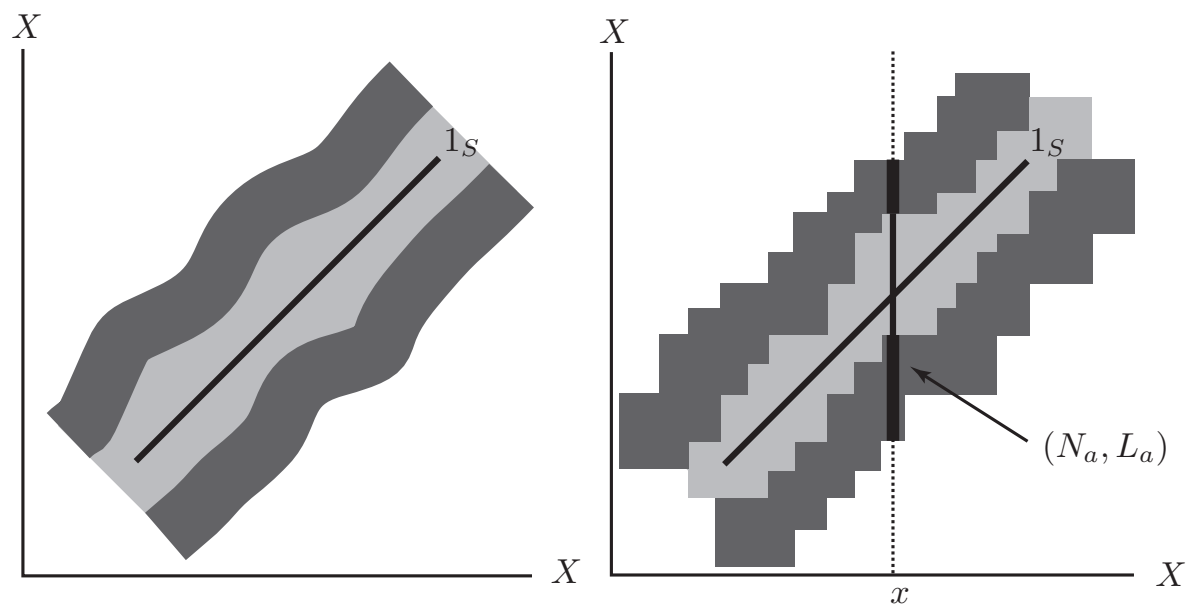

Figure 4. The given index pair for $1_{S}$, a discretized index pair nearby, and one of the finite number of slices.

Theorem 9 says that if $f$ is expansive on an isolated invariant set $S$, then it has an index system. Expansiveness is a strong condition that may be difficult to verify in practice. It turns out that a condition weaker than expansiveness guarantees the existence of index systems. In Theorem 9 we assumed that $1_{S} \subset X \times X$ was an isolated invariant set (i.e., that $f$ was expansive on $S$ ), but the technique for constructing the index system requires only that $1_{S}$ be contained in some isolated invariant set $\Lambda$. Then the slices of an index pair $(N, L)$ for $\Lambda$ give an index system for $S$. At one extreme we have $\Lambda=1_{S}$ ( $f$ is expansive), in which case we can find an index system with index pairs arbitrarily small. At the other extreme, for any isolated invariant set $S$ we could take $\Lambda=S \times S$; this would produce a trivial index system consisting of one index pair. There may be times when $1_{S} \subsetneq \Lambda \subsetneq S \times S$, and the resulting index system is useful. 
If $1_{S}$ is not an isolated invariant set, then we will not be able to make the index pairs arbitrarily small, but that is not necessarily a big disadvantage. On the one hand, the smaller the sets $\operatorname{cl}\left(N_{a} \backslash L_{a}\right)$, the stronger the conclusion of Theorem 7 . which is one reason the small diameters guaranteed by Theorem 9 are important. Furthermore, the smaller the sets, the more disjoint pairs we have, which, as we have seen, makes it easier to detect symbolic dynamics. On the other hand, small diameters can lead to a system with many small, individually unimportant pieces. This would produce a large graph, and thus a very complicated cocyclic subshift. So, in practice there is a trade-off involved in the size of the sets of the index system.

\section{EXAMPLES}

Example 10. Let $f: S^{1} \rightarrow S^{1}$ be the doubling map on the circle, considered as $\mathbb{R} / \mathbb{Z}$. The collection $\mathcal{P}=\left\{\left(N_{i}, L_{i}\right)\right\}_{i=0}^{9}$ is an index system, where $N_{i}=$ $\left[\frac{i-3-3 \varepsilon}{10}, \frac{i+3+3 \varepsilon}{10}\right]$ and $L_{i}=\left[\frac{i-3-3 \varepsilon}{10}, \frac{i-1-\varepsilon}{10}\right] \cup\left[\frac{i+1+\varepsilon}{10}, \frac{i+3+3 \varepsilon}{10}\right] . \quad P_{i}$ precedes $P_{j}$ for $j=2 i-1,2 i$, or $2 i+1 \bmod 10$ (see Figure 5 ).

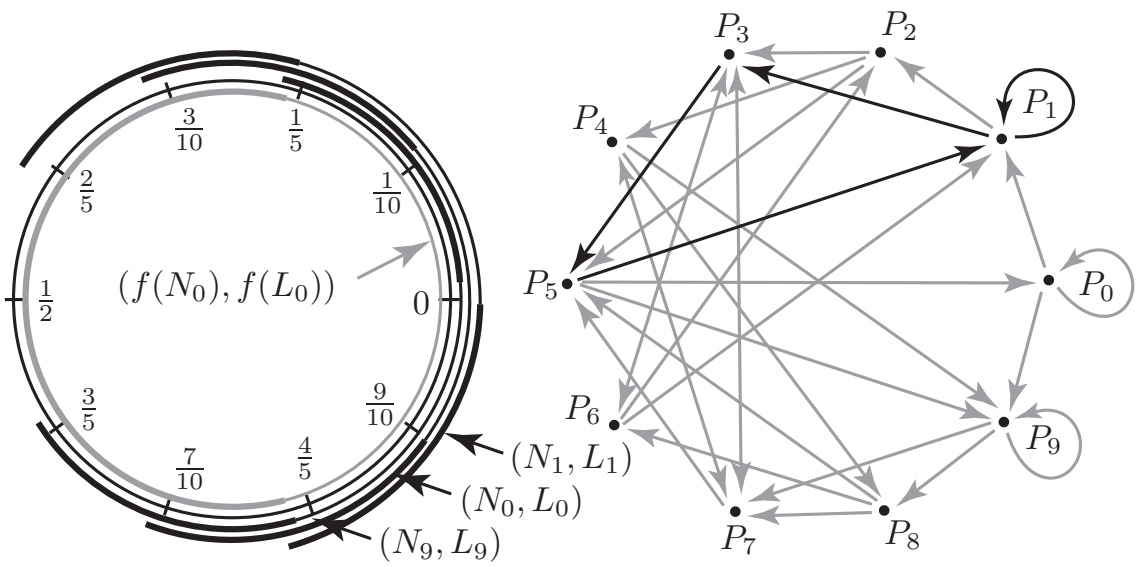

Figure 5. An example showing that $P_{0}$ precedes $P_{9}, P_{0}$, and $P_{1}$, and the directed graph for this index system.

Each pointed space $N_{i L_{i}}$ is homeomorphic to a circle. So, for every $i$, the only nonzero homology is $H_{1}\left(P_{i}\right)=\mathbb{Z}$, and the induced maps are $f_{i, j *}=1$ if $P_{i}$ precedes $P_{j}$, and $f_{i, j *}=0$ otherwise. Thus any concatenation of the maps $f_{(1,1,1) *}=f_{1,1 *} \circ$ $f_{1,1 *} \circ f_{1,1 *}$ and $f_{(1,3,5) *}=f_{5,1 *} \circ f_{3,5 *} \circ f_{1,3 *}$ is nonzero. Since the sets $\operatorname{cl}\left(\left(N_{1} \backslash L_{1}\right)\right)$ and $\operatorname{cl}\left(\left(N_{5} \backslash L_{5}\right)\right)$ are disjoint, so are the sets $S_{(1,1,1)}$ and $S_{(1,3,5)}$, and thus we see that the map $f^{3}: S_{(1,1,1)} \cup S_{(1,3,5)} \rightarrow S_{(1,1,1)} \cup S_{1,3,5}$ factors onto the full shift on two symbols. 
Example 11. Let $f: \mathbb{R} \rightarrow \mathbb{R}$ be the tent map given by

$$
f(x)=\left\{\begin{array}{l}
3 x, \text { if } x \leq 1 / 2, \\
3-3 x, \text { if } x \geq 1 / 2 .
\end{array}\right.
$$

The map $f \times f$ has an index pair shown in Figure 6 .

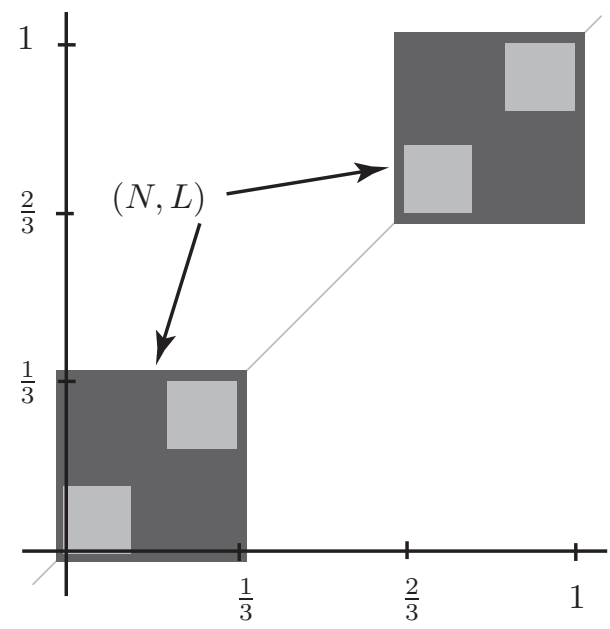

Figure 6 . An index pair for $f \times f$, where $f$ is the tent map.

Taking slices we obtain the index system $\mathcal{P}=\left\{\left(N_{i}, L_{i}\right)\right\}_{i=1}^{4}$ for $f$ (as shown in Figure 7), where

$$
\begin{aligned}
& N_{1}=N_{2}=\left[0-4 \varepsilon, \frac{1}{3}+4 \varepsilon\right], \\
& L_{1}=[0-4 \varepsilon, 0-\varepsilon] \cup\left[\frac{1}{9}+\varepsilon, \frac{1}{3}+4 \varepsilon\right], \\
& L_{2}=\left[0-4 \varepsilon, \frac{2}{9}-\varepsilon\right] \cup\left[\frac{1}{3}+\varepsilon, \frac{1}{3}+4 \varepsilon\right], \\
& N_{3}=N_{4}=\left[\frac{2}{3}-4 \varepsilon, 1+4 \varepsilon\right], \\
& L_{3}=\left[\frac{2}{3}-4 \varepsilon, \frac{2}{3}-\varepsilon\right] \cup\left[\frac{7}{9}+\varepsilon, 1+4 \varepsilon\right], \\
& L_{4}=\left[\frac{2}{3}-4 \varepsilon, \frac{8}{9}-\varepsilon\right] \cup[1+\varepsilon, 1+4 \varepsilon] .
\end{aligned}
$$

Again, each pointed space $N_{i L_{i}}$ is homeomorphic to a circle. $P_{1}$ and $P_{4}$ precede $P_{1}$ and $P_{2}$, while $P_{2}$ and $P_{3}$ precede $P_{3}$ and $P_{4}$. In homology, in dimension one, the induced maps are $f_{1,1 *}=f_{1,2 *}=f_{2,3 *}=f_{2,4 *}=1$ and $f_{3,3 *}=f_{3,4 *}=f_{4,1 *}=$ $f_{4,2 *}=-1$. Thus the tent map restricted to $\operatorname{Inv}[0-\varepsilon, 1+\varepsilon]$ factors onto the shift given by the graph in Figure 7 , which is conjugate to the full shift on two symbols $([18, \S 2.4])$.

Example 12. Let $f$ be the tent map from Example 11. The pair $\left(N_{0}, L_{0}\right)=$ $\left([-4 \varepsilon, 1+4 \varepsilon],[-4 \varepsilon,-\varepsilon] \cup\left[\frac{1}{3}+\varepsilon, \frac{2}{3}-\varepsilon\right] \cup[1+\varepsilon, 1+4 \varepsilon]\right)$ is an index pair, and thus also a (trivial) index system. The only nonzero homology is $H_{1}\left(N_{0}, L_{0}\right)=\mathbb{Z}^{2}$, and the induced map is given by $f_{00 *}=\left(\begin{array}{cc}1 & -1 \\ 1 & -1\end{array}\right)$. Since $\left(f_{00 *}\right)^{2}$ is the zero matrix, the cocyclic subshift is empty. Thus this index system fails to detect any invariant set. 

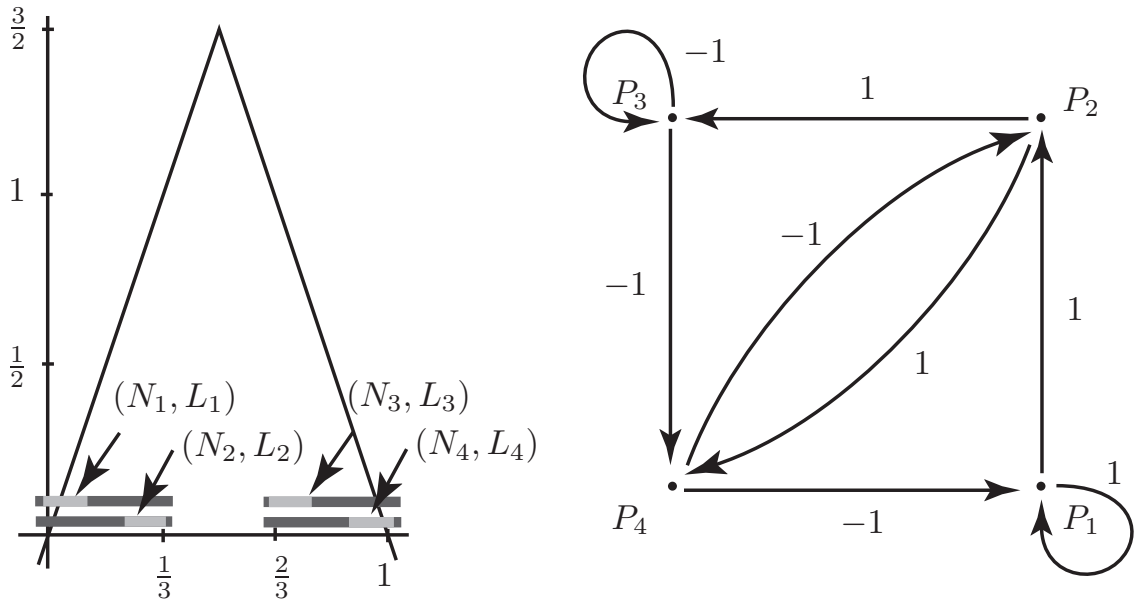

Figure 7. An index system for the tent map, and the associated directed graph.

\section{REFERENCES}

[1] E. Akin, The general topology of dynamical systems, American Mathematical Society, Providence, RI, 1993. MR.1219737(94f:58041)

[2] N. Aoki and K. Hiraide, Topological theory of dynamical systems, North-Holland Mathematical Library, vol. 52, North-Holland Publishing Co., Amsterdam, 1994. MR1289410 (95m:58095)

[3] G. Arioli, Periodic orbits, symbolic dynamics and topological entropy for the restricted 3-body problem, Comm. Math. Phys. 231 (2002), no. 1, 1-24. MR.1947690(2003j:70011)

[4] R. Bowen and J. Franks, Homology for zero-dimensional nonwandering sets, Ann. of Math. (2) 106 (1977), no. 1, 73-92. MR0458492 (56:16692)

[5] M. Carbinatto, J. Kwapisz, and K. Mischaikow, Horseshoes and the Conley index spectrum, Ergodic Theory Dynam. Systems 20 (2000), no. 2, 365-377. MR 1756975 (2001e:37020)

[6] S. Day, R. Frongillo, and R. Treviño, Algorithms for rigorous entropy bounds and symbolic dynamics, SIAM J. Appl. Dyn. Syst. 7 (2008), no. 4, 1477-1506. MR2470974(2010b:37023)

[7] S. Day, O. Junge, and K. Mischaikow, Towards automated chaos verification, Proceedings of EQUADIFF 2003, World Sci. Publ., Hackensack, NJ, 2005. 157-162. MR2185012

[8] R. Easton, Isolating blocks and symbolic dynamics, J. Differential Equations 17 (1975), 96118. MR0370663(51:6889)

[9] __ Orbit structure near trajectories biasymptotic to invariant tori, Classical mechanics and dynamical systems (Medford, Mass., 1979), Lecture Notes in Pure and Appl. Math., vol. 70, Dekker, New York, 1981, pp. 55-67. MR640118 (83h:58040)

[10] _ Isolating blocks and epsilon chains for maps, Phys. D 39 (1989), no. 1, 95-110. MR1021184 (90m:58176)

[11] J. Franks and D. Richeson, Shift equivalence and the Conley index, Trans. Amer. Math. Soc. 352 (2000), no. 7, 3305-3322. MR 1665329 (2000j:37013)

[12] M. Gîdea, Leray functor and orbital Conley index for non-invariant sets, Discrete Contin. Dynam. Systems 5 (1999), no. 3, 617-630. MR.1696333(2000f:37013)

[13] _ Non-smooth dynamical systems that exhibit hyperbolic behavior, Rev. Roumaine Math. Pures Appl. 45 (2000), no. 4, 631-646 (2001). MR1836294 (2002e:37014)

[14] M. Gîdea and C. Robinson, Symbolic dynamics for transition tori. II, New advances in celestial mechanics and Hamiltonian systems, Kluwer/Plenum, New York, 2004, pp. 95-108. MR2083007 (2005k:37130)

[15] B. Hasselblatt and A. Katok, Principal structures, Handbook of dynamical systems. Vol. 1A (B. Hasselblatt and A. Katok, eds.), North-Holland, Amsterdam, 2002, pp. 1-203. MR:1928517 (2003c:37002) 
[16] T. Kaczynski, Conley index for set-valued maps: from theory to computation, Conley index theory (Warsaw, 1997), Banach Center Publ., vol. 47, Polish Acad. Sci., Warsaw, 1999, pp. 57-65. MR1692361 (2000d:37015)

[17] J. Kwapisz, Cocyclic subshifts, Math. Z. 234 (2000), no. 2, 255-290. MR 1765882 (2001j:37025)

[18] D. Lind and B. Marcus, An introduction to symbolic dynamics and coding, Cambridge University Press, Cambridge, 1995. MR,1369092 (97a:58050)

[19] K. Mischaikow, Topological techniques for efficient rigorous computation in dynamics, Acta Numer. 11 (2002), 435-477. MR2009377 (2004k:37184)

[20] K. Mischaikow and M. Mrozek, Chaos in the Lorenz equations: a computer-assisted proof, Bull. Amer. Math. Soc. (N.S.) 32 (1995), no. 1, 66-72. MR1276767(95e:58121)

[21] M. Mrozek, Leray functor and cohomological Conley index for discrete dynamical systems, Trans. Amer. Math. Soc. 318 (1990), no. 1, 149-178. MR0968888 (90f:34076)

[22] _ The Conley index and rigorous numerics, Non-linear analysis and boundary value problems for ordinary differential equations (Udine), CISM Courses and Lectures, vol. 371, Springer, Vienna, 1996, pp. 175-195. MR1465243 (98i:58161)

[23] _ An algorithm approach to the Conley index theory, J. Dynam. Differential Equations 11 (1999), no. 4, 711-734. MR1725408 (2000j:37014)

[24] D. Richeson and J. Wiseman, Index systems and symbolic dynamics, in preparation.

[25] J. Robbin and D. Salamon, Dynamical systems, shape theory and the Conley index, Ergodic Theory Dynam. Systems 8* (1988), Charles Conley Memorial Issue, 375-393. MR0967645 (89h:58094)

[26] C. Robinson, Dynamical systems, Studies in Advanced Mathematics, CRC Press, Boca Raton, FL, 1995. MR $1396532(97 \mathrm{e}: 58064)$

[27] A. Szymczak, The Conley index for discrete semidynamical systems, Topology Appl. 66 (1995), no. 3, 215-240. MR1359514 (97f:58113)

[28] _. The Conley index and symbolic dynamics, Topology 35 (1996), no. 2, 287-299. MR 1380498 (97b:58054)

[29] J. B. van den Berg, R. C. Vandervorst, and W. Wójcik, Chaos in orientation reversing twist maps of the plane, Topology Appl. 154 (2007), no. 13, 2580-2606. MR.2332874(2008f:37093)

[30] J. Wiseman, Detection of renewal system factors via the Conley index, Trans. Amer. Math. Soc. 354 (2002), no. 12, 4953-4968 (electronic). MR.1926844(2003e:37022)

[31] — The square of a map, symbolic dynamics and the Conley index, Rocky Mountain J. Math. 37 (2007), no. 1, 327-342. MR2316452(2009b:37028)

$[32]$ K. Wójcik and P. Zgliczyński, Isolating segments, fixed point index, and symbolic dynamics. III. Applications, J. Differential Equations 183 (2002), no. 1, 262-278. MR1917245 (2003g:37023)

[33] P. Zgliczyński, Computer assisted proof of chaos in the Rössler equations and in the Hénon map, Nonlinearity 10 (1997), no. 1, 243-252. MR1430751(98g:58120)

[34] P. Zgliczyński and M. Gidea, Covering relations for multidimensional dynamical systems, J. Differential Equations 202 (2004), no. 1, 32-58. MR2060531(2005c:37019a)

Department of Mathematics and Computer Science, Dickinson College, Carlisle, Pennsylvania 17013

E-mail address: richesod@dickinson.edu

Department of Mathematics, Agnes Scott College, Decatur, Georgia 30030

E-mail address: jwiseman@agnesscott.edu 